\title{
Is the culture of medicine contributing to miscarriages among female physicians?
}

Cite as: CMAJ 2019 November 4;191:E1229-30. doi: 10.1503/cmaj.1095821

Posted on cmajnews.com on Oct. 15, 2019

W hen Dr. Ayesha Khan had a miscarriage after years of struggling with infertility, she didn't mention it to colleagues or miss a beat at work.

Khan, a clinical assistant professor in emergency medicine at Stanford University, now thinks that type of silence could be preventing pregnant doctors from understanding the potential risks of night shifts and long hours in early pregnancy and from seeking accommodations they may need.

At the recent FemInEM Idea Exchange conference in New York City, Khan and Dr. John Purakal, an emergency physician at Duke University, presented preliminary findings from a survey of 550 physicians, $51 \%$ of whom reported a miscarriage. Two-thirds of respondents said they were not aware of support systems, and none knew of accommodations for early pregnancy.

Most pregnant doctors wouldn't seek accommodations because, "there is very much this idea of, 'If you can't cut it, too bad,"' says Khan.

After the presentation, many doctors told Khan and Purakal their stories. One doctor said when her supervisor asked her to do a lumbar puncture on a patient with suspected meningitis, she didn't disclose her pregnancy for fear of being seen as a burden. Several physicians said they told no one of their miscarriages, even if they happened during work shifts.

The situation is similar in Canada. An Ontario doctor, who requested anonymity, told CMAJ that six years ago she was working about eight night-call shifts a month, sometimes up to 30 hours long, when she miscarried at 12 weeks. She told the supervisor of her pediatric fellowship and asked for fewer call shifts. "I was seen as a complainer," she says. Her schedule wasn't changed. Later, when she missed a presentation, for a dilation and curettage after a second miscarriage at nine weeks, "Others in the department questioned if I was just nervous to present and wanted to get out of it."

A review of 62 "low and very low" quality observational studies in the American Journal of Obstetrics and Gynecology found that working longer hours and night shifts were associated with a higher risk of miscarriage than standard, daytime hours. The review also found increased risks for preterm delivery, low birthweight, preeclampsia and hypertension among those who work night shifts and long hours.

One study, based on survey data for which the response rate was not known, found a rate of miscarriage among medical

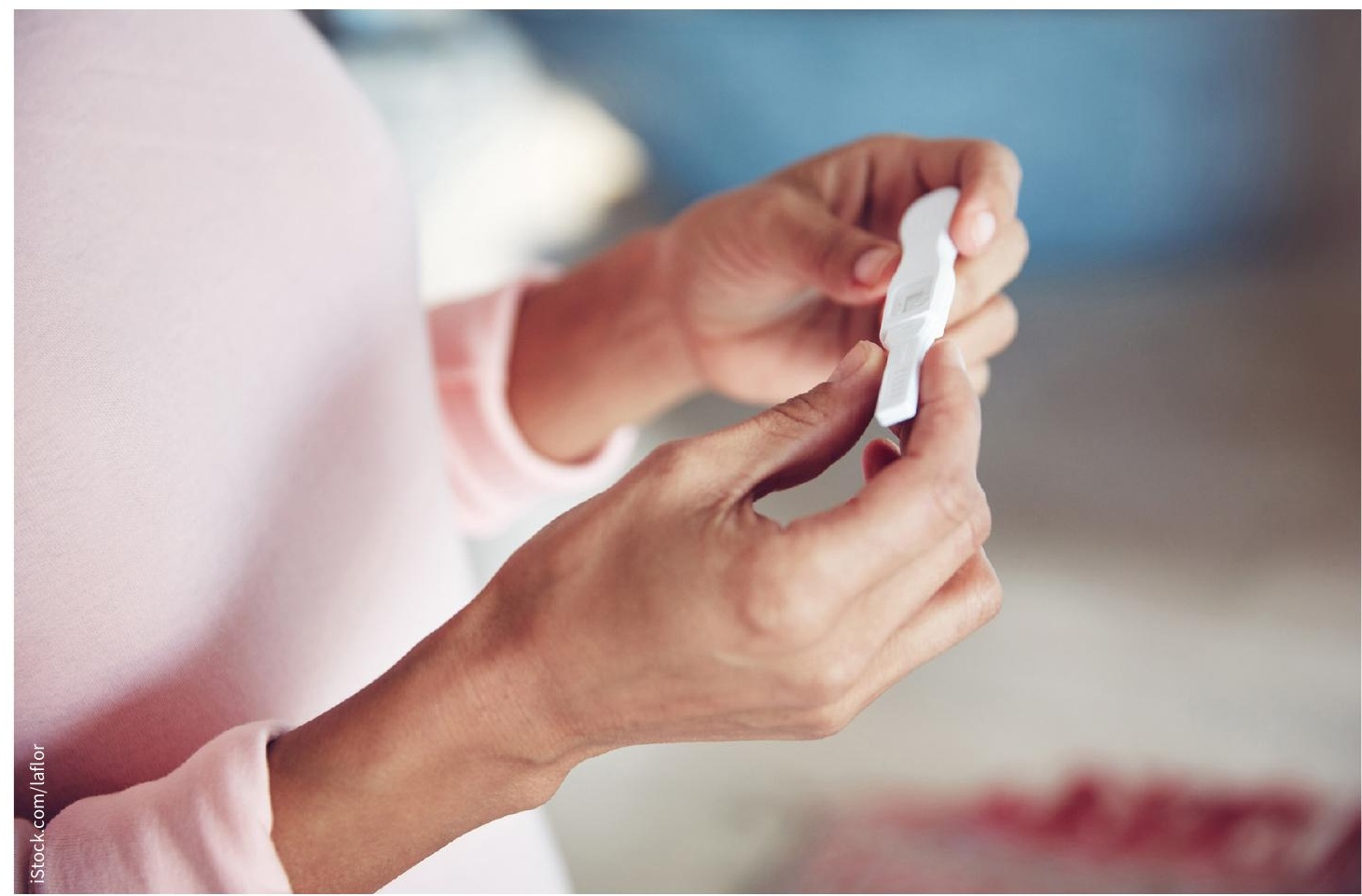

Some doctors are worried about the effects of long hours and night shifts on pregnancy. 
and surgical residents in North America to be almost three times higher than among general population controls. The rate of complications was lower among those who had six or fewer night call shifts per month (26\%) than among those who had more than six (49\%).

However, that research is "difficult to interpret," explains Dr. Fiona Mattatall, an obstetrician in Calgary who cares for pregnant residents. The study's findings were based on self-reported information on both workload and complications. As only 238 residents across 190 programs in North America responded, the study could be biased if those who experienced complications were more likely to do the survey.

Overall, the research on miscarriage and work hours and rotating shifts is weak, says Mattatall. The studies don't take into account other confounding variables, like socioeconomic status. Except for the survey, studies haven't targeted doctors, a population that is generally healthier but has children at an older age. She hopes a more robust study will be done. "We have enough female physicians and nurses that work night shifts that we can collect the information in a better way," she says.

Other physicians are also concerned and speaking out, often on social media, about how medicine can change to reduce the risk of miscarriage among pregnant physicians and better support those who experience miscarriage.

Dr. Kimi Chernoby, a chief resident in emergency medicine at Indiana University, thinks eliminating night call should be automatic when female physicians disclose pregnancy, although they could opt to do night call if they want. If physicians must request the accommodation, the culture of medicine is such that female physicians, especially in training, will feel pressured not to make the demand.

Chernoby forwarded a motion to her fellow emergency residents to take up additional night call so that pregnant residents can avoid those shifts. If it's successful, Chernoby plans to bring the vote to all residents at her school. Emergency medicine at her institution has 75 residents to pick up shifts but "with smaller programs, there is likely going to be more pushback."

Although pregnant doctors can make requests for accommodations, it doesn't always go well. When Dr. Kirstin Derdall was a pregnant resident, she started to feel contractions two weeks before she was protected from night call. She asked her supervisor to let her reduce her call shifts to 16 hours. She was accommodated, but some hospital staff made comments about her being "a princess" or asking if she had a good rest. "They were jokes," says Derdall, now an anesthesiologist and chronic pain specialist in Alberta, "but they weren't jokes."

In Canada, residents have some protection from night shifts in later pregnancy. In Quebec, residents aren't scheduled for night call starting at 20 weeks and in Manitoba, at 31 weeks. Dr. Jennifer Dvorak, a second-year anesthesiology resident at the University of Calgary, is asking the Resident Doctors of Canada to ensure night shift protection from 24 weeks or earlier across the country. Dvorak developed preeclampsia when pregnant with her son, who was born at 35 weeks because of intrauterine growth restriction. Her colleagues "were incredible" and encouraged her to take breaks, but she wonders how 24-hour call shifts affected her pregnancy. "You hardly have time to eat, and you're managing the ward and consults, and there's a lot of stress," she says.

Dvorak thinks residency agreements should list the tasks pregnant women are protected from, including procedures on a highly infectious patient. "That would be helpful to people who might not have the confidence to refuse work, and it also ensures everyone is on the same page."

Wendy Glauser, Toronto, Ont. 